\title{
On the Effect of Shear Loading Rate on Contact Area Shrinking in Adhesive Soft Contacts
}

\author{
Antonio Papangelo ${ }^{1,2}$ (1)
}

Received: 16 February 2021 / Accepted: 19 March 2021 / Published online: 2 April 2021

(c) The Author(s) 2021

\begin{abstract}
Adhesion and, its interplay with friction, is central in several engineering applications involving soft contacts. Recently, there has been an incredible push towards a better understanding on how the apparent contact area evolves when a shear load is applied to an adhesive soft contact, both experimentally and theoretically. Although soft materials are well-known to exhibit rate-dependent properties, there is still a lack of understanding in how the loading rate could affect the contact area shrinking. Indeed, most of the experiments involving a sphere-flat contact have been conducted at a fixed loading rate, and, so far, analytical models have assumed a constant work of adhesion, independent on the peeling velocity. Here, by using linear elastic fracture mechanics, an analytical model is derived for the contact of a rigid sphere on a soft adhesive substrate, which is aimed at elucidating the effect that a rate-dependent work of adhesion has on the contact area shrinking. The model results show that contact area reduction is very sensitive to the loading rate, with slower loading rates promoting a stronger shrinking, which seems in agreement with Literature results. Furthermore it is shown that rate effects enhance the apparent interfacial toughness, i.e. more energy is needed to drive the system from full stick up to gross sliding.
\end{abstract}

Keywords Adhesion $\cdot$ Soft matter $\cdot$ Contact area $\cdot$ Friction

\section{Introduction}

Adhesion is a flourishing area of tribology, which is central in both science and technology [1]. Thanks to evolution, Nature has developed several high efficient systems capable to control interfacial adhesion, as common experience proves when geckos, lizards and, in general, insects climb vertical walls or run on ceilings. Exploiting van der Walls forces in an efficient manner, is a primary scientific and technological task, particularly when it interferes with friction, as this has a tremendous impact on cutting edge technologies under development, such as soft robots [2], developing an artificial sense of touch [3], human-robot interactions [4], pressure-sensitive adhesives [5-7], grippers [8]. Recently, several authors have been working on the effect that a shear

Antonio Papangelo

antonio.papangelo@tuhh.de; antonio.papangelo@poliba.it

1 Department of Mechanics, Mathematics and Management, Politecnico di Bari, Via Orabona 4, 70125 Bari, Italy

2 Department of Mechanical Engineering, Hamburg University of Technology, Am Schwarzenberg-Campus 1, 21073 Hamburg, Germany force has on the reduction of contact area in a sphere-flat soft contact. According to the most recent contributions there are certainly two effects at play: (i) one is related to large deformations [9-11], (ii) the other related to the weakening effect that friction has on adhesion due to the mixed-mode type of loading the contact area is subjected to when a shearing load is applied [12-15]. Although a definite answer on the interaction between the two mechanisms is still lacking, using finite element simulations with coupled large deformation, adhesion and friction, Mergel et al. ([10], see their Fig. 13c) have shown that both effects (i) and (ii) are at play and concur to the reduction of contact area, with adhesion being determinant at low normal loads, while large deformations being more important for larger normal loads. Here we will restrict our attention to the range of lighter normal loads, where exploiting Linear Elastic Fracture Mechanics (LEFM) concepts, the contact area can be regarded as an external crack subjected to mixed-mode loading.

The basic idea of using LEFM for adhesive compliant bodies in contact, dates back to the seminal work of Johnson, Kendall and Roberts ([16], JKR in the following), who studied the normal contact of rubber (or gelatin) spheres. Savkoor and Briggs [12] further developed the work of 
Johnson et al. [16] to account for the possibility of a shearing load, which determines on the contact periphery mixedmode loading conditions. When compared with experiments, Savkoor and Briggs model [12] predicted a stronger reduction of contact area with respect to the one observed. Later on, various authors have contributed in extending the work of Savkoor and Briggs [12] and, in light of a better agreement with experiments, have introduced a phenomenological function, which accounted for the dependence of the interfacial toughness on the phase angle $\psi=\arctan \left(K_{\mathrm{II}} / K_{\mathrm{I}}\right)$, being $K_{\mathrm{II}}$ and $K_{\mathrm{I}}$ the Stress Intensity Factors respectively in mode II and mode I [13-15, 17-26]. The latter approach has some tradition in the fracture mechanics Literature and is supported by experimental evidences [27, 28], nevertheless it does not clarify the physical reasons behind the increase of interfacial toughness with the phase angle $\psi$.

Recently McMeeking et al. [15] have attempted a physically based interpretation of the experimental evidences. They have considered the possibility that, while the slip penetrates the contact area, the strain energy stored in interfacial defects such as dislocations or similar (see discussion in [18]), can be released, hence participating in the fracture process (i.e. weakening adhesion). Hence, only the reversible part (" $G_{\mathrm{II}}^{\text {rev") }}$ of the overall mode II energy release rate $G_{\text {II }}$, should be accounted when writing the Griffith energy balance

$G_{\mathrm{I}}+G_{\mathrm{II}}^{\mathrm{rev}}=w$,

where $G_{\mathrm{I}}$ is the mode I energy release rate, $G_{\mathrm{II}}^{\text {rev }}=\alpha G_{\mathrm{II}}$ being $\alpha$ a dimensionless shear-index that is postulated to be a constant and $w$ the work of adhesion (or surface energy). Ciavarella and Papangelo [24] have estimated the parameter $\alpha$ from different sets of experimental results available in Literature (particularly from: $[12,19,21,29]$ ), while Peng et al. [26] have measured $\alpha$ for both compliant and stiff spheres. Ciavarella and Papangelo [24] have found that $\alpha$ remained almost constant within a given experiment, suggesting that this may be an inherent property of the interface, but it seemed to be strongly dependent on the loading rate, when different experimental results were compared each other (see their Fig. 7).

Clearly experiments are challenging to run and most of the Literature results have been performed at a fixed loading rate within the same experiment, mostly trying to mimic the quasi-static loading conditions. Although, marked differences appear when one compares the experimental work of different authors. For example Savkoor and Briggs [12] applied the tangential load step-wise, with each step approximately equal to $\sim 2.5 \mathrm{mN}$ and with $1 \mathrm{~min}$ pause between one step and the other. Waters and Guduru [19] imposed the tangential load through a motorized stage actuated at a constant velocity of $0.5 \mu \mathrm{m} / \mathrm{s}$, Sahli et al. [21] and Mergel et al. [29] applied the tangential load through a loading arm actuated at $100 \mu \mathrm{m} / \mathrm{s}$ while Peng et al. [26] actuated the tangential stage at about $2 \mu \mathrm{m} / \mathrm{s}$. Clearly, adhesion measurements are very sensitive to details and it is difficult to compare results from different test rigs. Nevertheless Ciavarella and Papangelo [24] have shown that the parameter $\alpha$ seems to scale as a power law with the velocity at which the tangential loading stage is actuated. ${ }^{1}$

Although relevant steps forward have been made on the modeling side, the previously mentioned models have barely considered the effect that the tangential loading rate has on the contact area reduction, with the only exception of Waters and Guduru [19] who briefly discussed possible implications. Nevertheless, soft materials are well-known to exhibit rate-dependent behavior and it is widely accepted that the interfacial toughness shows a power law dependence on the peeling velocity $v_{\mathrm{p}}$, as firstly proposed by Gent and Schultz [30]

$w=w_{0}\left[1+\left(\frac{v_{\mathrm{p}}}{V_{0}}\right)^{n}\right]$

where $w_{0}$ is the adiabatic (or thermodynamic or Dupré) work of adhesion in the limit of $v_{\mathrm{p}} \rightarrow 0, V_{0}=\left(k a_{T}^{n}\right)^{-1}$ is a reference velocity, $k$ is a constant, $a_{T}$ is the WLF [31] shift factor that allows to compare viscoelastic data taken at different temperature and $n$ is an exponent commonly in a range from 0.1 to 0.9 for realistic materials.

In what follows, the model of Ciavarella and Papangelo [24] will be extended to account for a velocity dependent work of adhesion in the form of the Gent and Schultz law [Eq. (2)]. Hence, the presented model is solely based on linear elastic fracture mechanics and rate effects are limited to assuming a rate-dependent work of adhesion in the form of Eq. (2), while the study of the effect of substrate viscoelasticity is left to a further analysis.

\section{Theoretical Model}

\subsection{Dimensional Formulation}

Let us consider a rigid sphere of radius $R$ squeezed against a linear elastic soft half-space by a normal force $P$ and sheared by a tangential force $T$ (see Fig. 1). For soft materials we expect short-range adhesion, hence in the normal direction the classical JKR model is considered. In the tangential direction we assume that, as the shear force $T$ is increased, slip starts to penetrate within the contact circle, determining a slip annulus $b<r \leq a$, where $r$ is the radial coordinate,

\footnotetext{
$\overline{1}$ The parameter $\alpha$ is referred as $\lambda$ in [24].
} 
Fig. 1 a Sketch of the contact problem considered. A rigid sphere is pressed and sheared on a soft elastic half-space. b The periphery of the contact area is subjected to mixed-mode fracture loading. The shaded inner circle of radius $b$ represents the stick region, while the contact area has radius $a$ (a) Sketch of the problem considered

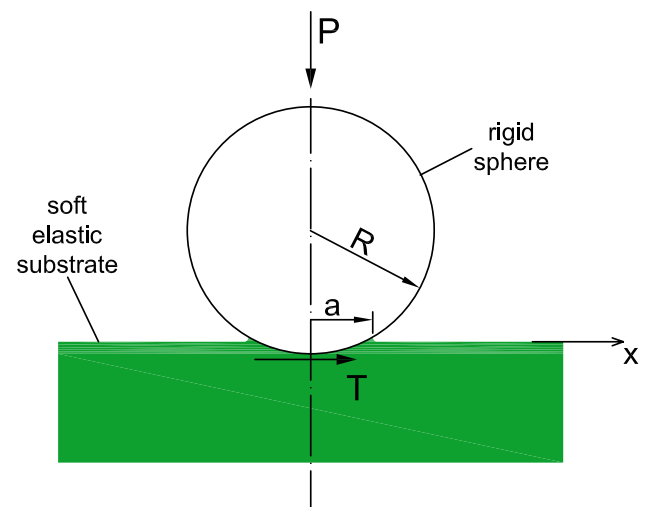

(b) Fracture modes combination

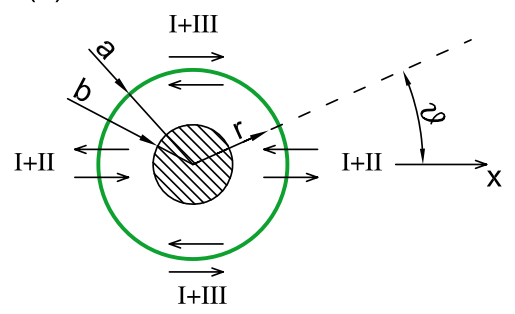

$a$ is the contact radius and $b$ is the radius of the stick zone (Fig. 1b). As suggested by experimental observations [25, 32,33 , within the slip annulus a uniform constant shear traction $\tau_{0}$ is assumed, which resultant equilibrates the external force $T$.

From the classical JKR theory, the mode I energy release rate is

$G_{\mathrm{I}}=\frac{1}{8 \pi E^{*} a^{3}}\left(\frac{4 E^{*} a^{3}}{3 R}-P\right)^{2}$,

where $E^{*}=E /(1-v)^{2}$ is the plain strain elastic modulus of the substrate, with $E$ the Young's modulus and $v$ the Poisson ratio. In the tangential direction, using the equivalent of a Dugdale crack in mode II and the definition of $\alpha$, the reversible mode II energy release rate is

$G_{\mathrm{II}}^{\mathrm{rev}}=\alpha G_{\mathrm{II}}=\alpha \tau_{0} \bar{\delta}_{0}$,

where $\bar{\delta}_{0}$ is the total slip averaged at the contact periphery [34]

$\bar{\delta}_{0}=\frac{2}{\pi}\left(\frac{2-v}{1-v}\right) \frac{\tau_{0}}{E^{*}} a\left(\sqrt{1-\left(\frac{b}{a}\right)^{2}} \cos ^{-1}\left(\frac{b}{a}\right)+\frac{b}{a}-1\right)$.

Furthermore, from Savkoor PhD thesis [34] the tangential load $T$ and the tangential remote displacement $D$ are respectively

$D=\frac{2-v}{1-v} \frac{\tau_{0}}{E^{*}} a \sqrt{1-\left(\frac{b}{a}\right)^{2}}$,
$T=2 \tau_{0} a^{2}\left(\frac{b}{a} \sqrt{1-\left(\frac{b}{a}\right)^{2}}+\cos ^{-1}\left(\frac{b}{a}\right)\right)$.

Using the results in [15], Ciavarella and Papangelo [24] have shown that $\bar{\delta}_{0}$ can be expressed, exactly, as a function of the tangential load $T$ as

$\bar{\delta}_{0}=\bar{\delta}_{0, \mathrm{LEFM}} h\left(\frac{T}{T_{\text {full }}}\right)=\frac{(2-v)}{2(1-v)} \frac{T^{2}}{8 \pi \tau_{0} E^{*} a^{3}} h\left(\frac{T}{T_{\text {full }}}\right)$,

where $\bar{\delta}_{0, \mathrm{LEFM}}$ is the averaged slip at the contact periphery in the LEFM limit, $T_{\text {full }}=\tau_{0} \pi a^{2}$ and $h\left(T / T_{\text {full }}\right)$ is a parametric corrective function (see [24] for further details).

Using Eq. (1) the Griffith energy balance gives

$\frac{1}{8 \pi E^{*} a^{3}}\left(\frac{4 E^{*} a^{3}}{3 R}-P\right)^{2}+\alpha \tau_{0} \bar{\delta}_{0, \mathrm{LEFM}} h\left(\frac{T}{T_{\text {full }}}\right)=w$.

If the work of adhesion does not depend on the peeling velocity and is equal to its adiabatic value $\left(w=w_{0}\right)$, Eq. (9) coincides with the model presented in [24]. Instead, according to the Gent and Schultz law (2), here it is assumed that $w$ is a function of the peeling velocity

$v_{\mathrm{p}}=-\frac{\mathrm{d} a}{\mathrm{~d} t}=-\frac{\mathrm{d} a}{\mathrm{~d} T} \frac{\mathrm{d} T}{\mathrm{~d} t}=-\frac{\mathrm{d} a}{\mathrm{~d} T} T$,

where $t$ is the time, $T=\mathrm{d} T / \mathrm{d} t$ is the tangential loading rate and the minus sign accounts that for increasing $T$ the contact patch shrinks ( $\mathrm{d} a / \mathrm{d} t<0$ for $T>0$ ). ${ }^{2}$ Finally, using Eqs. (2), (8), (9), (10), the Griffith equilibrium condition gives

$\frac{\mathrm{d} a}{\mathrm{~d} T}=-\left(\frac{V_{0}}{\dot{T}}\right)\left\{\left[\left(P-\frac{4 E^{*} a^{3}}{3 R}\right)^{2}+\frac{1}{2}\left(\frac{2-v}{1-v}\right) \alpha T^{2} h\left(\frac{T}{T_{\text {full }}}\right)\right] \frac{1}{8 \pi a^{3} E^{*} w_{0}}-1\right\}^{1 / n}$,

2. One may also express the peeling velocity as a function of $D=\mathrm{d} D / \mathrm{d} t$. For this case, we have derived the governing differential equation in "Appendix". 
which is a first order differential equation that can be solved using as initial condition a contact radius that satisfies the JKR model under 0 tangential load, i.e.

$P=\frac{4 E^{*} a^{3}}{3 R}-\sqrt{8 \pi a^{3} E^{*} w_{0}}$.

From Eq. (9) one can obtain the equivalent work of adhesion

$w_{\mathrm{e}}=w_{0}\left(1+\frac{1}{V_{0}^{n}}\left(-\frac{\mathrm{d} a}{\mathrm{~d} T} \dot{T}\right)^{n}\right)-\frac{\alpha}{2}\left(\frac{2-v}{1-v}\right) \frac{T^{2}}{8 \pi a^{3} E^{*}} h\left(\frac{T}{T_{\text {full }}}\right)$.

Notice that, as $w_{\mathrm{e}}$ increases with the loading rate $\dot{T}_{T}$, the contact area is expected to shrink faster for low loading rates. This is in agreement with the analysis of Ciavarella and Papangelo [24] that suggests $\alpha$ is a decreasing function of the loading rate, i.e. a stronger reduction should be obtained in quasi-static conditions.

\subsection{Dimensionless Formulation}

Let us introduce the following dimensionless quantities

$$
\begin{aligned}
& \xi=\left(\frac{E^{*} R}{w_{0}}\right)^{1 / 3} ; \quad \tilde{a}=\frac{\xi a}{R} ; \quad \widetilde{T}=\frac{T}{R w_{0}} ; \quad \widetilde{v}_{\mathrm{p}}=\frac{v_{\mathrm{p}}}{V_{0}} ; \\
& \widetilde{w}_{\mathrm{e}}=\frac{w_{\mathrm{e}}}{w_{0}} ; \quad \widetilde{P}=\frac{P}{R w_{0}} ; \quad \dot{T}=\frac{T}{V_{0} \xi w_{0}} ; \quad \widetilde{\tau}_{0}=\frac{\tau_{0} R}{\xi^{2} w_{0}} .
\end{aligned}
$$

The differential equation (11) reduces to

$$
\frac{d \widetilde{a}}{d \widetilde{T}}=-\frac{1}{\widetilde{T}}\left\{\left[\left(\widetilde{P}-\frac{4}{3} \widetilde{a}^{3}\right)^{2}+\frac{\alpha}{2}\left(\frac{2-v}{1-v}\right) \widetilde{T}^{2} h\left(\frac{\widetilde{T}}{\widetilde{T}_{\text {full }}}\right)\right] \frac{1}{8 \pi \widetilde{a}^{3}}-1\right\}^{1 / n} .
$$

With the above notation the JKR equilibrium equation gives

$$
\widetilde{P}=\frac{4}{3} \widetilde{a}^{3}-\sqrt{8 \pi \widetilde{a}^{3}},
$$

while the equivalent surface energy is

$$
\widetilde{w}_{\mathrm{e}}=\left(1+\left(-\frac{\mathrm{d} \widetilde{a}}{\mathrm{~d} \widetilde{T}} \dot{\widetilde{T}}\right)^{n}\right)-\frac{\alpha}{2}\left(\frac{2-v}{1-v}\right) \frac{\widetilde{T}^{2}}{8 \pi \widetilde{a}^{3}} h\left(\frac{\widetilde{T}}{\widetilde{T}_{\text {full }}}\right)
$$

\section{Results}

\subsection{Contact Area Shrinking}

In what follows, we will focus on the effects that a ratedependent work of adhesion has on the reduction of contact area under increasing tangential load. As for the discussion above, unless differently stated, we will set $\alpha=1$. For a fixed set of the system parameters, reducing $\alpha$ would give qualitatively similar results except for a smaller reduction of the contact $\operatorname{area}^{3}$ (the smaller $\alpha$ the smaller $G_{\mathrm{II}}^{\text {rev }}$ available in the fracture process). Furthermore, our attention will be

\footnotetext{
$\overline{3}$ We neglect here the unlikely case of having $\alpha<0$.
} 

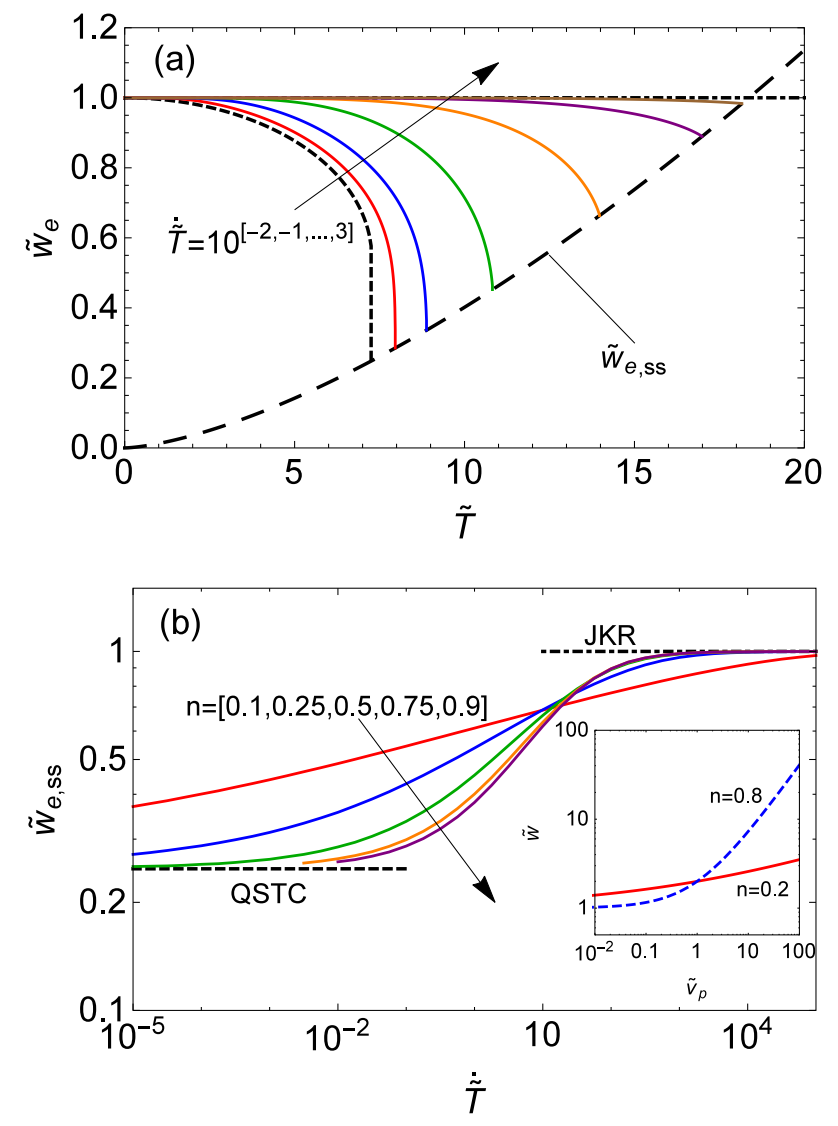

Fig. 3 a Equivalent surface energy $\widetilde{w}_{\mathrm{e}}$ as a function of the tangential load $\widetilde{T}$ for the curves in Fig. $2 \mathrm{a}\left(n=0.5, \log _{10} \widetilde{T}=[-2,-1, \ldots, 3]\right)$. b Equivalent adhesive energy $\widetilde{w}_{\text {e,ss }}$ at the point of incipient gross sliding as a function of the loading rate $\widetilde{T}$ for $n=[0.1,0.25,0.5,0.75,0.9]$. (Inset) Work of adhesion $\widetilde{w}$ as a function of the peeling velocity $\widetilde{v}_{p}$ for $n=0.2$ (solid curve) and $n=0.8$ dashed curve

restricted to the case of small normal loads, as to avoid nonlinear effects due to large deformations, hence, in the following, unless differently stated, we will set $\widetilde{P}=0$.

Let us first investigate the reduction of contact area as a function of the tangential load $\widetilde{T}$. Figure 2 a shows the variation of contact area $\widetilde{A}=\pi \widetilde{a}^{2}$ as a function of the tangential load $\widetilde{T}$ for $n=0.5, \widetilde{\tau}_{0}=1$ and different loading rates $\log _{10} \widetilde{T}=[-2,-1, \ldots, 3]$ (solid curves). Increasing the tangential load $\widetilde{T}$ the contact area shrinks, more rapidly as the loading rate reduces. The dot-dashed black curve, corresponds to the Quasi-STatiC (QSTC) limit, where $w=w_{0}$, while the dashed gray line represents the condition $\widetilde{T}=\widetilde{\tau}_{0} \widetilde{A}$, i.e. the state of incipient gross sliding. Panel (b) sheds a light on the effect of the exponent $n$ on the contact area reduction at a fixed loading rate $\tilde{T}=1$. It is shown that $n$ has a strong effect on the rate at which the contact area shrinks, in particular larger $n$ leads to a stronger reduction of the contact

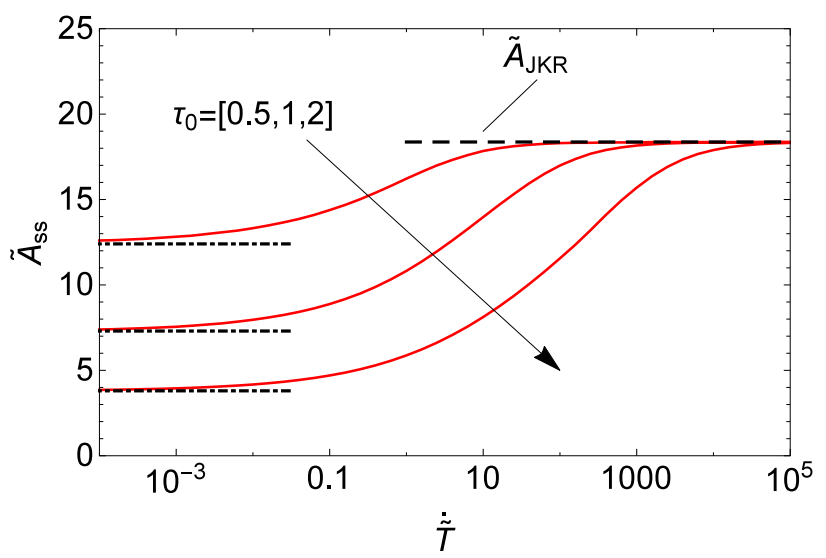

Fig. 4 The ratio $\widetilde{T}_{\text {ss }} / \widetilde{\tau}_{0}$ as a function of $\dot{\widetilde{T}}$ for $n=0.5$ and varying $\tilde{\tau}_{0}=[0.5,1,2]$.

area. This can be easily understood by looking at how the equivalent energy $w_{\mathrm{e}}$ varies during the shrinking process.

Figure 3 a shows the equivalent adhesive energy $\widetilde{w}_{\mathrm{e}}$ as a function of the tangential load $\widetilde{T}$ for the curves in Fig. $2 \mathrm{a}$ $\left(n=0.5, \log _{10} \widetilde{T}=[-2,-1, \ldots, 3]\right)$. For vanishing tangential load $\widetilde{T}$ all the curves start at $\widetilde{w}_{\mathrm{e}}=1$, i.e. from the JKR solution. While the tangential load is increased, the equivalent adhesive energy diminishes up to the critical condition of incipient gross sliding, to which we refer as $\widetilde{w}_{\mathrm{e}, \mathrm{ss}}$. The limit curve for the equivalent adhesion energy in the instant of incipient gross sliding $\widetilde{w}_{\mathrm{e}, \mathrm{ss}}$ (long dashed black line) can be easily obtained substituting $\widetilde{a}=\left(\frac{\widetilde{T}}{\pi \widetilde{\tau}_{0}}\right)^{1 / 2}$ into Eq. (17). Figure 3 a shows that the curves for $\widetilde{w}_{\mathrm{e}}$ (solid curves) are bounded by the QSTC limit (short dashed black line), which gives the fastest reduction of the equivalent surface energy, and the limit case of infinite loading rate (horizontal dot-dashed line), where $\widetilde{w}_{\mathrm{e}}$ remains constant, as the contact area does not have time to shrink.

To gain a better understanding of the effect of the loading rate on the contact area shrinking, it is useful to show how the equivalent surface energy at the instant of incipient gross sliding $\widetilde{w}_{\mathrm{e}, \mathrm{ss}}$ varies as a function of the loading rate and of the exponent $n$. Figure $3 \mathrm{~b}$ shows $\widetilde{w}_{\text {e,ss }}$ versus $\dot{T}$ for $n=[0.1,0.25,0.5,0.75,0.9]$. First notice that all the curves start from the QSTC value at low $\dot{\widetilde{T}}$ and tend to 1 at high loading rates. It is shown that all the curves intersect at $\widetilde{T} \approx 10$. In particular for $\widetilde{T} \lesssim 10$ a small exponent $n$ gives a higher equivalent surface energy, while for $\widetilde{T} \gtrsim 10$ a small exponent $n$ gives a lower equivalent surface energy. This depends on the shape of the Gent and Schultz law (2). Indeed, the inset shows the dimensionless work of adhesion $\widetilde{w}$ as a function of the dimensionless peeling velocity 
$\widetilde{v}_{\mathrm{p}}=v_{\mathrm{p}} / V_{0}$ for $n=0.2$ (solid curve) and $n=0.8$ (dashed curve). Clearly, in the range of $\widetilde{v}_{\mathrm{p}}<1$, a smaller exponent gives a larger adhesive energy, hence, when the peeling process starts with a low peeling velocity $\left(\widetilde{v}_{\mathrm{p}}<1\right)$, a slower reduction of the contact area is obtained (see Fig. 2b).

Finally Fig. 4 shows the contact area at incipient gross sliding $\widetilde{A}_{\text {ss }}$, as a function of the loading rate $\tilde{\widetilde{T}}$ for $n=0.5$ and varying $\widetilde{\tau}_{0}=[0.5,1,2]$ (solid curves). The dot-dashed black lines stand for $\widetilde{A}_{\mathrm{ss}}$ for QSTC loading, while the upper limit at high loading rates is the JKR contact area. For high loading rates all the curves tend to the JKR contact area, regardless of the shearing strength $\widetilde{\tau}_{0}$, while the QSTC limit is strongly influenced by $\widetilde{\tau}_{0}$. As one may expect, the larger $\widetilde{\tau}_{0}$ the larger the reduction of the contact area that can be achieved in the QSTC conditions.

\subsection{Rate-Dependent Toughening of the Interface}

Here the toughening effect that a rate-dependent work of adhesion has on the contact interface is investigated. To allow for a quantitative comparison, it is convenient to determine the amount of mechanical energy $E_{\mathrm{t}}$ that has to be spent to drive the system from full-stick to the state of incipient gross sliding. The energy $E_{\mathrm{t}}$ spent in the transition can be computed as

$E_{\mathrm{t}}=\int_{0}^{D_{\text {full }}} T \mathrm{~d} D=\int_{1}^{0} T(\widetilde{b}) \frac{\mathrm{d} D(\widetilde{b})}{\mathrm{d} \widetilde{b}} \mathrm{~d} \widetilde{b}$,

where $D_{\text {full }}=\frac{(2-\nu)}{(1-\nu)} \frac{\tau_{0} a}{E^{*}}$ is the remote displacement at the state of incipient gross sliding and we have highlighted that the tangential load $T$ and displacement $D$ are function of $\widetilde{b}=b / a$. Using Eq. (6) to obtain the derivative $\mathrm{d} D / \mathrm{d} \widetilde{b}$ and substituting the latter into Eq. (18) one obtains in dimensionless form

$\widetilde{E}_{\mathrm{t}}=2\left(\frac{2-v}{1-v}\right) \widetilde{\tau}_{0}^{2} \int_{0}^{1} \frac{\left(\widetilde{b} \sqrt{1-\widetilde{b}^{2}}+\cos ^{-1}(\widetilde{b})\right) \widetilde{b}}{\sqrt{1-\widetilde{b}^{2}}} \widetilde{a}^{3} d \widetilde{b}$,

where $\widetilde{E}_{\mathrm{t}}=E_{\mathrm{t}} \xi^{2} /\left(w_{0} R^{2}\right)$. In general, the reduction rate of the contact radius $\widetilde{a}$ will be governed by the differential equation (15), hence it will not be a trivial function of $\widetilde{b}$. Nevertheless Eq. (15) suggests that for very high loading rates $\mathrm{d} \widetilde{a} / \mathrm{d} \widetilde{T} \rightarrow 0$, hence, in the transition from sticking to sliding, the contact radius will not have time to shrink and will remain approximately constant and equal to its JKR value, i.e. $\tilde{a} \approx \tilde{a}_{\mathrm{JKR}}$. This allows to determine an upperbound for the transition energy $\widetilde{E}_{\mathrm{t}, \max }$ (in the limit of very high loading rate) that is

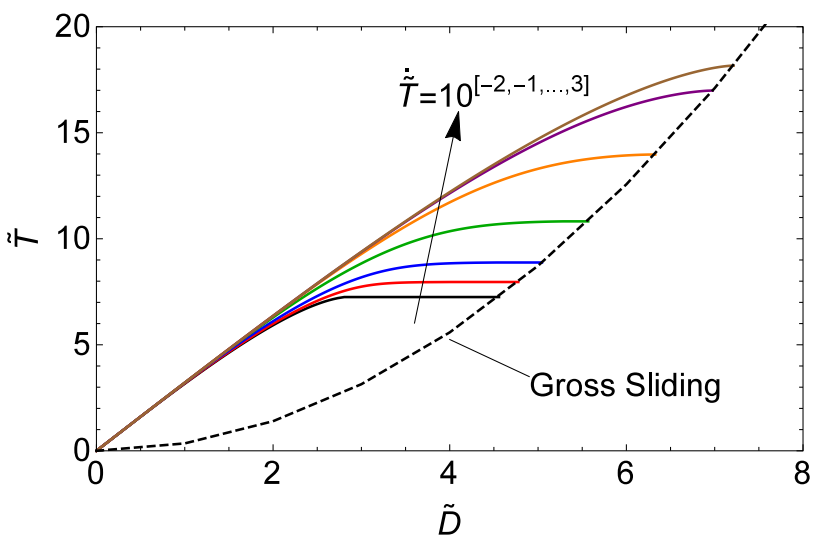

Fig. 5 Tangential load versus the dimensionless tangential remote displacement $\widetilde{D}$ for different loading rates $\log _{10} \widetilde{T}=[-2,-1, \ldots, 3]$ and $n=0.5, \widetilde{\tau}_{0}=1$. Same parameters as in Fig. $2 \mathrm{a}$

$\widetilde{E}_{\mathrm{t}, \max }=\left(\pi-\frac{4}{3}\right)\left(\frac{2-v}{1-v}\right) \widetilde{\tau}_{0}^{2} \widetilde{a}_{\mathrm{JKR}}^{3}$.

For the curves plotted in Fig. 2a, Fig. 5 shows the dimensionless tangential load versus the dimensionless tangential remote displacement $\widetilde{D}=\frac{D E^{*}}{\xi w_{0}}$ for different loading rates $\log _{10} \widetilde{T}=[-2,-1, \ldots, 3]$ and $n=0.5, \widetilde{\tau}_{0}=1$. All the curves start linearly from the origin, as in the very beginning the contact radius remains almost constant, and bend horizontally when the contact area shrinks markedly. A larger loading rates leads to a larger tangential force and displacement at incipient gross sliding, which from Eqs. (6), (7) is $\widetilde{T}_{\mathrm{ss}}=\frac{\pi \widetilde{D}^{2}}{\widetilde{\tau}_{0}\left(\frac{2-v}{1-v}\right)^{2}}$. As the energy $\widetilde{E}_{\mathrm{t}}$ spent in the transition from full-stick to gross sliding is the area underneath the loading curve, it is evident that high loading rates make the interface tougher.

In order to quantitatively compare the toughening effect introduced by a rate-dependent work of adhesion, Fig. 6a plots the energy $\widetilde{E}_{\mathrm{t}}$ as a function of the loading rate for $n=0.5$ and $\widetilde{\tau}_{0}=[0.5,1,2]$. First note that $\widetilde{E}_{\mathrm{t}}$ increases with the interfacial shear strength $\widetilde{\tau}_{0}$, with a sigmoidal shape that starts off at low loading rate from the QSTC value $\widetilde{E}_{\text {qstc }}$ and increases up to $\widetilde{E}_{\mathrm{t}, \max }$ at high $\dot{\widetilde{T}}$ (both limits are shown as dashed black lines). Figure $6 \mathrm{~b}$ shows that the ratio $\widetilde{E}_{\mathrm{t}} / \widetilde{E}_{\mathrm{qstc}}$ is strongly affected by $\tilde{T}$ and is larger for higher $\widetilde{\tau}_{0}$. This suggests possible new avenues of research for tough interfaces in soft materials, where the loading rate may play a fundamental role.

\subsection{Scaling of the Shear-Index $\alpha$}

As we have reviewed in the Introduction, few experimental data are available in Literature to compare with, as different experimentalists have used different protocols of actuation 
Fig. 6 a The energy $\widetilde{E}_{\mathrm{t}}$ as a function of the loading rate $\tilde{T}$ for $n=0.5$ and $\widetilde{\tau}_{0}=[0.5,1,2]$. Dashed black lines refer to the QSTC and the high loading rate limits [Eq. (20)]. b For the curve in panel $\mathbf{a}$, panel $\mathbf{b}$ shows that the ratio $\widetilde{E}_{\mathrm{t}} / \widetilde{E}_{\mathrm{qstc}}$, being $\widetilde{E}_{\mathrm{qstc}}$ the energy spent in the transition from sticking to incipient sliding in QSTC conditions

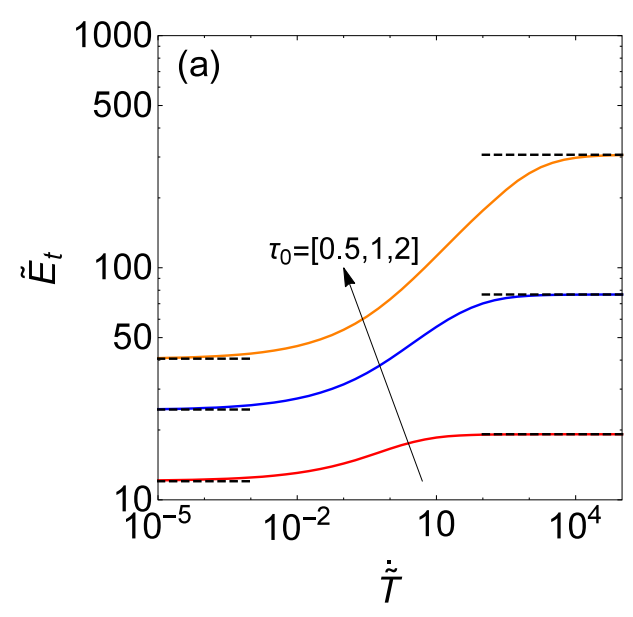

of the tangential loading stage. Nevertheless, collecting the data available, Ciavarella and Papangelo [24] have shown that the shear-index $\alpha$ is expected to scale as a power law with the velocity $v_{\mathrm{s}}$ at which the tangential loading arm is actuated-more precisely they found $\alpha \propto v_{\mathrm{s}}^{-0.8}$. In line with Waters and Guduru analysis [19], Ciavarella and Papangelo [24] speculated that this scaling may be compatible with rate-dependent effects, which were absent in their model.

Although a quantitative comparison with experimental results is not possible for lacking information, we here attempt to derive what would be the scaling of the shear-

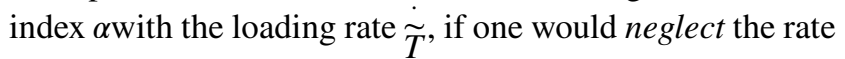
dependence of the work of adhesion on the peeling velocity, as indeed most of the authors have done so far. Using $w=w_{0}$, from Eq. (9) one obtains in dimensionless form

$\alpha_{\mathrm{fit}}=\frac{8 \pi \widetilde{a}^{3}-\left(\frac{4}{3} \widetilde{a}^{3}-\widetilde{P}\right)^{2}}{\frac{3}{2} \widetilde{T}^{2} h\left(\frac{\widetilde{T}}{\pi \widetilde{\tau}_{0} \widetilde{a}^{2}}\right)}$

which can be exploited to determine how $\alpha_{\text {fit }}$ depends on $\dot{\tilde{T}}$. Hence, the objective, is to determine which $\alpha_{\text {fit }}$ would better fit the result obtained with a rate-dependent model [Eq. (15)], $\widetilde{a}$ versus $\widetilde{T}$ curve, if one assumes the work of adhesion is constant $\left(w=w_{0}\right)$. To this end we fixed a given exponent $n=[0.2,0.3,0.4,0.5,0.8]$, then solved the differential equation (15) to obtain the curve $\widetilde{a}$ versus $\widetilde{T}$ and determined $\alpha_{\text {fit }}$ imposing that Eq. (21) is satisfied at $\widetilde{T}=7$. We have verified that the scaling of $\alpha_{\text {fit }}$ with the loading rate is not influenced by this arbitrary choice of $\widetilde{T}$. For the curve $\widetilde{a}$ versus $\widetilde{T}$ obtained with the rate-dependent model [Eq. (15)] in Fig. 2a corresponding to $\widetilde{T}=10^{-1}$ (blue solid line), with the above procedure, one obtains that it can be approximately fitted by a model with constant work of adhesion $\left(w=w_{0}\right)$ but using $\alpha_{\mathrm{fit}}=0.6637$ (and not $\alpha=1$ as in the rate-dependent model), which gives the purple dashed line shown in Fig. 2a.
Figure 7 shows the results obtained for the exponents $n=[0.2,0.3,0.4,0.5,0.8]$. At low loading rates all the curves tend to $\alpha_{\text {fit }}=1$, i.e. in QSTC conditions rate effects can be neglected and both the elastic and the rate-dependent models give the same shear-index $\left(\alpha=\alpha_{\mathrm{fit}}\right)$. It has been shown before that larger $\tilde{\widetilde{T}}$ leads to a smaller reduction of the contact area, hence $\alpha_{\text {fit }}$ starts to decrease as a power law. For all the exponents considered we found $\alpha_{\mathrm{fit}} \propto \tilde{\widetilde{T}}^{-1}$, which slope is quite close to the "-0.8" reported in [24]. The mechanism behind is clear: to favor a slower reduction of the contact area $\alpha_{\text {fit }}$ decreases, i.e. it decreases the reversible part of the mode II energy release available in the fracture process. In light of recent experimental measurements/estimation of the shear-index $\alpha[24,26]$ and to achieve a better understanding of the physical meaning of the shear-index $\alpha$, it is of utmost importance to discern between rate-dependent effects and the irreversible

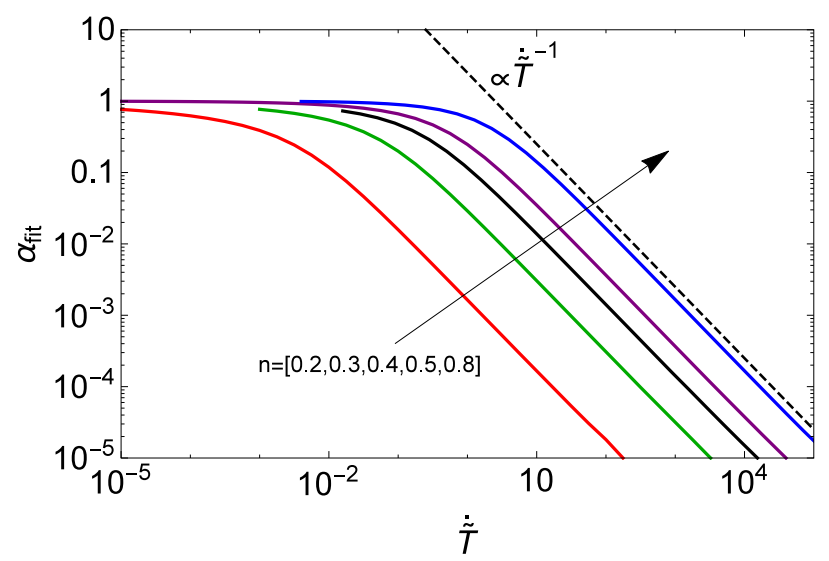

Fig. 7 Shear-index $\alpha_{\mathrm{fji}}$ obtained fitting the rate-dependent model results' ( $\widetilde{a}$ versus $\widetilde{T}$ curves) with an elastic model with constant work of adhesion, as a function of the loading rate $\widetilde{T}$ for $n=[0.2,0.3,0.4,0.5,0.8]$ 
processes happening at the interface. Notice that, in this analysis we have neglected bulk effects (we used the relaxed modulus of the viscoelastic material $E_{0}=E(\omega=0)$, being $\omega$ the excitation frequency), hence the results presented should be relied on for not too high peeling velocity.

\section{Conclusions}

In this manuscript the contact between a rigid sphere, pressed against a elastic soft substrate and sheared by a tangential force, has been considered. The effect that a velocity dependent work of adhesion (in the form of the Gent and Schultz law) has on the reduction of the contact area, have been studied and discussed. It has been shown that the tangential loading rate has a strong impact on the rate at which the contact area shrinks, in particular stronger reduction is predicted for QSTC loading conditions, while at a high tangential loading rate, the contact circle does not have time to shrink and the contact area remains almost constant, equal to its JKR value. Furthermore, a high loading rate makes the interface tougher, in the sense that more energy has to be spent to drive the system form full stick to the condition of incipient gross sliding. The toughening effect can be quite strong, particularly for high interface shear strength. Finally, following recent emphases on measuring the shearindex $\alpha$, it has been shown that neglecting the dependence of the work of adhesion on the peeling velocity, may lead to and erroneous estimate of $\alpha$, which may appear to scale as a power law of the loading rate. This is in agreement with the estimate of Ciavarella and Papangelo [24]. Nevertheless, a quantitative comparison with experimental results was out of reach as, commonly, sphere-flat shear experiments have been conducted at a fixed loading rate, hiding possible ratedependent effects. With the current emphasis in assessing if the shear-index $\alpha$ is a property of the interface $[15,24,26]$ or just a "fitting" parameter with a blurry physical meaning, it is of utmost importance for experimentalists to investigate more on the effect that the loading velocity has on the contact area shrinking and to discern rate effects from the irreversible processes happening at the interface, which should be captured by $\alpha$. In this respect, it is hoped that the present model would be of help.

\section{Appendix}

In the main text, we have expressed the peeling velocity as a function of the loading rate $T=\mathrm{d} T / \mathrm{d} t$, which is the rate of change of the tangential load with respect to time, measured in SI units in (N/s). It may be useful, for further analysis, to express the peeling velocity as a function of the imposed velocity at which the remote tangential displacement is applied, i.e. $D=\mathrm{d} D / \mathrm{d} t$ measured in SI units in $(\mathrm{m} / \mathrm{s})$. The peeling velocity is then

$v_{\mathrm{p}}=-\frac{\mathrm{d} a}{\mathrm{~d} t}=-\frac{\mathrm{d} a}{\mathrm{~d} D} \frac{\mathrm{d} D}{\mathrm{~d} t}=-\frac{\mathrm{d} a}{\mathrm{~d} D} \dot{D}$,

where the minus sign accounts that for increasing $D$ the contact patch shrinks (d $a / \mathrm{d} t<0$ for $D>0)$. From Savkoor [34] the total slip averaged at the contact periphery is

$\bar{\delta}_{0}=\frac{2}{\pi} D g(\bar{D})$,

where $\bar{D}=D / D_{\text {full }}$ and $g(\bar{D})$ is the function

$g(\bar{D})=\frac{\left(\sqrt{1-\bar{D}^{2}}+\bar{D} \sin ^{-1}(\bar{D})-1\right)}{\bar{D}}$.

Using Eqs. (1), (2), (3), (4), (22), (23), (24), the Griffith equilibrium condition gives

$\frac{\mathrm{d} a}{\mathrm{~d} D}=-\left(\frac{V_{0}}{\dot{D}}\right)\left[\frac{1}{8 \pi E^{*} a^{3} w_{0}}\left[\left(\frac{4 E^{*} a^{3}}{3 R}-P\right)^{2}+16 \alpha E^{*} a^{3} \tau_{0} D g\left(\frac{D}{D_{\text {full }}}\right)\right]-1\right]^{1 / n}$

which is a first order differential equation that can be solved using as initial condition a contact radius that satisfies the JKR equation.

Author Contributions AP is responsible for all the work.

Funding Open access funding provided by Politecnico di Bari within the CRUI-CARE Agreement.. A.P. acknowledges the support by the Italian Ministry of Education, University and Research under the Programme Department of Excellence Legge 232/2016 (Grant No. CUPD94I18000260001). A.P. is thankful to the DFG (German Research Foundation) for funding the Project PA 3303/1-1. A.P. acknowledges support from "PON Ricerca e Innovazione 2014-2020-Azione I.2" - D.D. n. 407, 27/02/2018, bando AIM (Grant No. AIM1895471).

Data Availability All data available upon request at the author's email address.

Code Availability Custom code available upon request at the author's email address.

\section{Declarations}

Conflict of interest AP declare no conflict of interest.

Open Access This article is licensed under a Creative Commons Attribution 4.0 International License, which permits use, sharing, adaptation, distribution and reproduction in any medium or format, as long as you give appropriate credit to the original author(s) and the source, provide a link to the Creative Commons licence, and indicate if changes were made. The images or other third party material in this article are 
included in the article's Creative Commons licence, unless indicated otherwise in a credit line to the material. If material is not included in the article's Creative Commons licence and your intended use is not permitted by statutory regulation or exceeds the permitted use, you will need to obtain permission directly from the copyright holder. To view a copy of this licence, visit http://creativecommons.org/licenses/by/4.0/.

\section{References}

1. Ciavarella, M., Joe, J., Papangelo, A., Barber, J.R.: The role of adhesion in contact mechanics. J. R. Soc. Interface 16(151), 20180738 (2019)

2. Rus, D., Tolley, M.T.: Design, fabrication and control of soft robots. Nature 521(7553), 467-475 (2015)

3. Bartolozzi, C., Natale, L., Nori, F., Metta, G.: Robots with a sense of touch. Nat. Mater. 15(9), 921 (2016)

4. Wang, H., Totaro, M., Beccai, L.: Toward perceptive soft robots: progress and challenges. Adv. Sci. 5(9), 1800541 (2018)

5. McMeeking, R.M., Ma, L., Arzt, E.: Bi-stable adhesion of a surface with a dimple. Adv. Eng. Mater. 12(5), 389-397 (2010)

6. Papangelo, A., Ciavarella, M.: A Maugis-Dugdale cohesive solution for adhesion of a surface with a dimple. J. R. Soc. Interface 14(127), 20160996 (2017)

7. Papangelo, A., Ciavarella, M.: Adhesion of surfaces with wavy roughness and a shallow depression. Mech. Mater. 118, 11-16 (2018)

8. Li, L., Liu, Z., Zhou, M., Li, X., Meng, Y., Tian, Y.: Flexible adhesion control by modulating backing stiffness based on jamming of granular materials. Smart Mater. Struct. 28(11), 115023 (2019)

9. Lengiewicz, J., de Souza, M., Lahmar, M.A., Courbon, C., Dalmas, D., Stupkiewicz, S., Scheibert, J.: Finite deformations govern the anisotropic shear-induced area reduction of soft elastic contacts. J. Mech. Phys. Solids 143, 104056 (2020)

10. Mergel, J.C., Scheibert, J., Sauer, R.A.: Contact with coupled adhesion and friction: computational framework, applications, and new insights. J. Mech. Phys. Solids 146, 104194 (2021)

11. Wang, J., Tiwari, A., Sivebaek, I.M., Persson, B.N.J.: Sphere and cylinder contact mechanics during slip. J. Mech. Phys. Solids 143, 104094 (2020)

12. Savkoor, A.R., Briggs, G.A.D.: The effect of tangential force on the contact of elastic solids in adhesion. Proc. R. Soc. Lond. A 356(1684), 103-114 (1977)

13. Papangelo, A., Ciavarella, M.: On mixed-mode fracture mechanics models for contact area reduction under shear load in soft materials. J. Mech. Phys. Solids 124, 159-171 (2019)

14. Sahli, R., Pallares, G., Papangelo, A., Ciavarella, M., Ducottet, C., Ponthus, N., Scheibert, J.: Shear-induced anisotropy in rough elastomer contact. Phys. Rev. Lett. 122(21), 214301 (2019)

15. McMeeking, R.M., Ciavarella, M., Cricri, G., Kim, K.S.: The interaction of frictional slip and adhesion for a stiff sphere on a compliant substrate. J. Appl. Mech. 87(3), 031016 (2020)

16. Johnson, K.L., Kendall, K., Roberts, A.D.: Surface energy and the contact of elastic solids. Proc. R. Soc. A 324, 301-313 (1971)

17. Johnson, K.L.: Continuum mechanics modeling of adhesion and friction. Langmuir 12, 4510-4513 (1996)
18. Johnson, K.L.: Adhesion and friction between a smooth elastic spherical asperity and a plane surface. Proc. R. Soc. Lond. A453(1956), 163-179 (1997)

19. Waters, J.F., Guduru, P.R.: Mode-mixity-dependent adhesive contact of a sphere on a plane surface. Proc. R. Soc. A 466(2117), 1303-1325 (2010)

20. Ciavarella, M.: Fracture mechanics simple calculations to explain small reduction of the real contact area under shear. Facta univ. ser. mech. eng. 16(1), 87-91 (2018)

21. Sahli, R., Pallares, G., Ducottet, C., Ben Ali, I.E., Al Akhrass, S., Guibert, M., Scheibert, J.: Evolution of real contact area under shear. Proc. Natl Acad. Sci. USA 115(3), 471-476 (2018)

22. Papangelo, A., Scheibert, J., Sahli, R., Pallares, G., Ciavarella, M.: Shear-induced contact area anisotropy explained by a fracture mechanics model. Phys. Rev. E 99(5), 053005 (2019)

23. Papangelo, A., Cricr, G., Ciavarella, M.: On the effect of the loading apparatus stiffness on the equilibrium and stability of soft adhesive contacts under shear loads. J. Mech. Phys. Solids 144, 104099 (2020)

24. Ciavarella, M., Papangelo, A.: On the degree of irreversibility of friction in sheared soft adhesive contacts. Tribol. Lett. 68(3), 1-9 (2020)

25. Das, D., Chasiotis, I.: Sliding of adhesive nanoscale polymer contacts. J. Mech. Phys. Solids (2020). https://doi.org/10.1016/j.jmps.2020. 103931

26. Peng, B., Li, Q., Feng, X.Q., Gao, H.: Effect of shear stress on adhesive contact with a generalized Maugis-Dugdale cohesive zone model. J. Mech. Phys. Solids 148, 104275 (2020)

27. Hutchinson, J.W.: Mixed mode fracture mechanics of interfaces. In: Rühle, M., Evans, A.G., Ashby, M.F., Hirth, J.P. (eds.) Metal ceramic interfaces, vol. 4, pp. 295-306. Pergamon Press, Oxford (1990)

28. Hutchinson, J.W., Suo, Z.: Mixed mode cracking in layered materials. In: Hutchinson, J.W., Wu, T.Y. (eds.) Advances in Applied Mechanics, vol. 29, pp. 63-191. Academic, Boston (1992)

29. Mergel, J.C., Sahli, R., Scheibert, J., Sauer, R.A.: Continuum contact models for coupled adhesion and friction. J. Adhes. 95(12), 11011133 (2019)

30. Gent, A.N., Schultz, J.: Effect of wetting liquids on the strength of adhesion of viscoelastic material. J. Adhes. 3(4), 281-294 (1972). https://doi.org/10.1080/00218467208072199

31. Williams, M.L., Landel, R.F., Ferry, J.D.: The temperature dependence of relaxation mechanisms in amorphous polymers and other glass-forming liquids. J. Am. Chem. Soc. 77, 3701-3707 (1955). https://doi.org/10.1021/ja01619a008

32. Carpick, R.W., Agrait, N., Ogletree, D.F., Salmeron, M.: Variation of the interfacial shear strength and adhesion of a nanometer-sized contact. Langmuir 12(13), 3334-3340 (1996)

33. Chateauminois, A., Fretigny, C.: Local friction at a sliding interface between an elastomer and a rigid spherical probe. Eur. Phys. J 27(2), 221-227 (2008)

34. Savkoor, A.R.: Dry adhesive friction of elastomers: a study of the fundamental mechanical aspects. PhD Dissertation, Technical University of Delft, The Netherlands (1987)

Publisher's Note Springer Nature remains neutral with regard to jurisdictional claims in published maps and institutional affiliations. 